\title{
Sobrepeso entre adolescentes de escolas particulares de Fortaleza, CE, Brasil
}

\author{
Overweight among adolescents from private schools in Fortaleza, CE, Brazil \\ Sobrepeso entre adolescentes de escuelas privadas de Fortaleza, CE, Brasil
}

\section{Márcio Flávio Moura Araújo', Lívia Silva de Almeida', Poliana Carina Viana da Silva', Hérica Cristina Alves de Vasconcelos', Marcos Venícius de Oliveira Lopes', Marta Maria Coelho Damasceno' \\ 'Universidade Federal do Ceará. Programa de Pós-graduação em Enfermagem. Fortaleza, CE}

Submissão: 01/06/2009

Aprovação: 09/07/2010

\section{RESUMO}

O objetivo da pesquisa foi identificar casos de excesso de peso em adolescentes de escolas particulares de Fortaleza - Brasil. Foram investigados 794 estudantes entre 12 e 17 anos de idade, de 12 escolas privadas, nos meses de março-junho e agosto-setembro de 2007. A prevalência de sobrepeso e obesidade foi, respectivamente, de $20 \%$ e $4 \%$. O sobrepeso foi maior, e duplicado os casos de obesidade, naqueles que mantinham o hábito de assistir tevê durante as refeições $(p=0,000)$. Cerca de $28 \%$ dos jovens ativos apresentavam excesso de peso $(p<0,001)$. No combate à adiposidade juvenil, cabe a enfermagem, principalmente, realizar as ações preventivas, pois atenuam diversas repercussões patológicas, relacionados com o acúmulo de peso corporal.

Descritores: Sobrepeso, Obesidade, Saúde do adolescente.

\section{ABSTRACT}

The purpose of the study was to identify cases of overweight among adolescents in private schools in Fortaleza, Brazil. We investigated 794 students between 12 and 17 years old from 12 private schools, in the months March to lune and August-September 2007. The prevalence of overweight and obesity was $20 \%$ and $4 \%$, respectively. Among the young people who watch TV while eat, the weight accumulated was higher and the cases of obesity was the double $(p=0,000)$. About $28 \%$ of people who practiced regular physical activity were overweight ( $<<0,00 \mathrm{I}$ ). In the fight against adolescent adiposity, to the nursing is primarily the preventive actions, because attenuate various pathological effects related to the accumulation of body weight.

Key words: Overweight; Obesity; Adolescent health.

\section{RESUMEN}

El objetivo de la investigación fue identificar los casos de sobrepeso en adolescentes de escuelas privadas de Fortaleza-Brasil. Fueron investigados 794 estudiantes entre 12 y 17 años, de 12 escuelas privadas, en los meses de marzo-junio y agosto-septiembre de 2007. La prevalencia de sobrepeso y obsedidad fue de $20 \%$ y $4 \%$, respectivamente. La acumulación de peso fue mayor y los casos de obesidad fue dos veces más, en los Que tenían la costumbre de asistir a la tele al alimentarse $(p=0,000)$. Sobre $28 \%$ de los jóvenes Que practicaban actividad física regularmente presentaban sobrepeso $(\mathrm{p}<0,001)$. Para la enfermería luchar contra la obesidad entre adolescentes, son necesarias las acciones preventivas, pues atenuan los diversos efectos patológicos con relación a la acumulación de peso corporal.

Descriptores: Sobrepeso; Obesidad; Salud del adolescente.

AUTOR CORRESPONDENTE Márcio Flávio Moura Araújo. Rua Conselheiro da Silva, 708. Barroso. CEP 60862-610. Fortaleza, CE.

E-mail: marciofma@yahoo.com.br 


\section{INTRODUÇÃO}

O atual estilo de vida globalizado trouxe aos países em desenvolvimento uma inversão epidemiológica: a decadência das deficiências nutricionais, doenças infecciosas e a ascensão das doenças crônico-degenerativas, especialmente as cardiovasculares. Tal fato pode estar relacionado às alterações da dieta e da composição corporal dos indivíduos ${ }^{(1-2)}$.

Conforme as estatísticas revelam, durante os últimos 25 anos a obesidade tornou-se um problema de saúde coletiva na América Latina. Acompanhando tendências globais, verifica-se Que o sobrepeso e a obesidade constituem, atualmente, o distúrbio nutricional Que mais cresce no continente latino. Especificamente entre a população abaixo de 20 anos de idade este vem assumindo proporções de uma epidemia ${ }^{(1-3)}$.

A prevalência da adiposidade juvenil ainda não é conhecida exatamente, porém, segundo se estima, um Quinto da população mundial entre 10 e 19 anos é acometida por acúmulo de peso No Brasil, conforme as estimativas, de sobrepeso e obesidade ocorrem entre $13,9 \%$ e $7,7 \%$, respectivamente, nos adolescentes ${ }^{(4)}$. Outros dados estabelecem também Que a prevalência de adiposidade triplicou, em detrimento da redução pela metade dos casos de desnutrição no $\mathrm{Brasil}^{(3)}$.

Desse modo, dentro de 20 anos, se medidas não forem tomadas, o país estará na atual situação na atual situação dos Estados Unidos, onde a obesidade e suas complicações constituem um dos maiores problemas de saúde pública, atingindo cerca de $26 \%$ dos jovens ${ }^{(1)}$.

Sobretudo, nas classes menos favorecidas da sociedade brasileira vive-se uma vertiginosa progressão dos casos de acúmulo de peso juvenil. Na cidade de Fortaleza, uma das maiores do Brasil, por exemplo, o último estudo desenvolvido no tema foi em escolas públicas, com jovens de 10 a 19 anos, e detectou uma taxa de cerca de $13 \%$ para o excesso de peso ${ }^{(5)}$. lá uma publicação anterior desenvolvida com adolescentes de escolas públicas e particulares de Fortaleza, detectou uma prevalência total de sobrepeso e obesidade de 19,5\%, sendo Que nas escolas privadas, sobrepeso/ obesidade alcançou 23,9\%, frequência maior do Que nas públicas $(18,0 \%)^{(6)}$.

Mesmo assim, entre os adolescentes de melhor condição socioeconômica, inseridos em escolas privadas, ainda há poucas investigações sobre esse assunto com amostras e análise representativas. $\mathrm{O}$ Que pode ser creditado às dificuldades e restrições impostas pelos responsáveis por essas populações e/ou instituições para a execução de peşuisas.

A obesidade, na infância e na adolescência, tem sido associada ao surgimento de morbidades como a hipertensão, principal doença cardiovascular de países em transição epidemiológica como o Brasil, dislipidemias, apnéia do sono, problemas ortopédicos, distúrbios emocionais e Diabetes Mellitus tipo 2 (DM 2) na adultícia ou ainda na própria infância e/ou adolescência. Em países desenvolvidos, a obesidade também tem sido relacionada a um menor grau de sucesso profissional e a menores salários ${ }^{(7-8)}$.

Diante da ascensão da prevalência do sobrepeso e da obesidade entre os jovens, somada à carência de publicações desenvolvidas com adolescentes de Fortaleza-Brasil, inseridos em famílias de melhor condição socioeconômica, verificou-se ser relevante identificar casos de sobrepeso e obesidade entre adolescentes de escolas particulares em Fortaleza - Brasil.

\section{MÉTODO}

Trata-se de um estudo transversal, cujos dados foram extraídos de um banco de dados construído por ocasião do desenvolvimento da pesQuisa "Identificação dos fatores de risco para diabetes mellitus tipo 2 em adolescentes". Referida investigação foi realizada em 12 estabelecimentos de ensino da rede privada, situados na cidade de Fortaleza - Brasil. Segundo informações do Centro Regional de Desenvolvimento da Educação (CREDE), Fortaleza é dividida em seis regiões, e, em março de 2007, possuía um total de 69.74 I alunos matriculados nas escolas particulares, na faixa etária de interesse dessa investigação (12-17 anos), distribuídos nas regiões, como segue na Tabela 1.

Para o cálculo da amostra utilizou-se uma fórmula para população infinita, a saber: $\mathrm{n}=\mathrm{t}^{2}{ }_{5 \%} \times \mathrm{P} \times \mathrm{Q} / \mathrm{e}^{2}$. Onde $\mathrm{t}=$ valor $\mathrm{da}$ distribuição de Student $\left(\mathrm{t}_{5 \%}=1,96\right) ; \mathrm{P}=50 \% ; \mathrm{Q}=100-\mathrm{P}=50 \%$; $\mathrm{e}=$ erro amostral absoluto $=4 \%$. Considerou-se os valores supracitados para P e Q haja vista proporcionarem um tamanho máximo de amostra, além do nível de significância $(a ́=0,05)$ e o erro amostral absoluto de $4 \%$. Assim, o número de participantes determinado foi equivalente a 600 adolescentes. Em virtude das prováveis perdas de sujeitos e/ou de informações, acrescentou-se uma margem de $20 \%$ a esse tamanho amostral. Desse modo, o "n" final abrangeu 720 estudantes (Tabela 1).

A proposta metodológica era de cada uma das seis regiões, escolher por conveniência duas escolas, localizadas em bairros distintos e com diferentes cenários de infra-estrutura e condições socioeconômicas, com vistas a se construir um panorama representativo da cidade de Fortaleza. Contudo, depois ela teve Que ser alterada, pois, muitos diretores escolares recusaram a realização da pesQuisa em suas respectivas instituições, alegando como empecilho a exposição dos alunos, o desenvolvimento de pesquisas anteriores sem retorno para a escola, além da retirada dos alunos da sala de aula, principalmente daqueles que estavam no ensino médio, em preparação para o vestibular.

Dessa forma, foi necessária a modificação da proposta inicial, com a participação de apenas uma escola nas regiões 1 e 5 e Quatro escolas na região 2. Neste último caso, apenas duas escolas não foram suficientes para contemplar a amostra estipulada para a região 2 Que era maior, sendo de 363 alunos. Portanto, a investigação ao seu final envolveu 794 alunos e 12 escolas, ou

Tabela 1. Estratificação da amostra dos adolescentes de escolas particulares, segundo a divisão por regional. Fortaleza-CE, 2007.

\begin{tabular}{lcc}
\hline Região & $\begin{array}{c}\text { No de alunos matriculados na } \\
\text { faixa etária de interesse }\end{array}$ & $\begin{array}{c}\text { Amostra por } \\
\text { região }\end{array}$ \\
\hline 1 & 5.510 & 51 \\
2 & 30.215 & 363 \\
3 & 7.101 & 61 \\
4 & 12.539 & 131 \\
5 & 5.602 & 36 \\
6 & 8.774 & 78 \\
\hline TOTAL & $\mathbf{6 9 . 7 4 1}$ & 720 \\
\hline
\end{tabular}


seja, cerca de $12 \%$ de todos os adolescentes das escolas particulares de Fortaleza na faixa de interesse da pesquisa daquele período.

Quanto à seleção dos sujeitos, deu-se de forma aleatória simples, por sorteio, entre os Que concordaram em participar da pesquisa e apresentaram o termo de consentimento livre e esclarecido, devidamente assinado tanto por eles como por seus pais ou responsáveis. Antes, porém, diretores, professores e alunos receberam informações sobre os objetivos e a metodologia da investigação.

Como critérios adotados para a seleção dos alunos avaliados mencionam-se os seguintes: 1- Ter entre 12-17 anos de idade; 2. Ser estudante dos turnos matutino/vespertino, período em Que se coletavam os dados; 3- Não apresentar QualQuer condição patológica ou outra passível de interferir na mensuração dos dados antropométricos (peso e altura).

A opção de identificar casos de sobrepeso/obesidade na faixa etária ora citada, ocorreu em congruência com as disposições de outros estudos, segundo os Quais, a ascensão de casos de adiposidade situa-se nessa faixa etária ${ }^{(7-8)}$. A princípio, houve interesse em se avaliar jovens de 18 anos de idade, porém, conforme percebido já na fase de seleção das escolas, esta faixa dificilmente seria encontrada, visto Que nas escolas privadas os alunos concluem tanto o ensino fundamental Quanto o médio na faixa etária prevista pelas leis de diretrizes e bases para educação, ou seja, antes dos 18 anos de idade.

A coleta de dados ocorreu em dois momentos, durante marçojunho e agosto-setembro de 2007. Envolveu entrevistas mediante a utilização de um formulário com Questões acerca das características sociodemográficas, além da mensuração do peso e da altura para o cálculo do Índice de Massa Corporal (IMC), obtido pela fórmula [peso $(\mathrm{kg})$ /altura $\left(\mathrm{m}^{2}\right)$ ].

Os dados antropométricos (peso e altura) foram mensurados uma única vez mediante alguns cuidados. O peso foi obtido com os jovens descalços e com roupas leves, por meio do uso de uma balança portátil digital com capacidade para $150 \mathrm{~kg}$ e uma precisão de $0,1 \mathrm{~kg}$. A estatura foi verificada a partir de uma fita métrica com escala de $0,5 \mathrm{~cm}$. A fim de assegurar a precisão da estatura, os pesquisados foram orientados a se posicionarem eretos e imóveis, com as mãos espalmadas sobre as coxas e com a cabeça ajustada ao plano de Frankfurt ${ }^{(9)}$.

Durante a análise, especificamente, do estado nutricional dos adolescentes avaliados, via IMC, adotou-se como critério de classificação preconizado por Cole e colaboradores (2000), referenciados em diversas publicações nacionais e internacionais. Eles desenvolveram parâmetros para sobrepeso e obesidade em meninas e meninos de 12 a 18 anos, a partir da relação entre IMC, idade e sexo, baseando-se em estudos transversais representativos de seis países, dentre eles o Brasil, cada um com mais de 10 mil participantes. Os pontos de corte foram ajustados de forma Que os percentis 85 e 95 de IMC aos 18 anos fossem obrigatoriamente os mesmos para sobrepeso e obesidade utilizados respectivamente para adultos $\left(25 \text { e } 30 \mathrm{~kg} / \mathrm{m}^{2}\right)^{(10)}$.

A medida da glicemia capilar foi realizada e os resultados obtidos foram analisados conforme indicadores do Ministério da Saúde do Brasil. Dessa forma, os valores foram interpretados, como seguem: menores Que $140 \mathrm{mg} / \mathrm{dl}$, normal, entre 141 e $199 \mathrm{mg} / \mathrm{dl}$, duvidoso, entre 200 e $270 \mathrm{mg} / \mathrm{dl}$ provável diabetes e, maior ou igual a 270mg/ dl, muito provável diabetes ${ }^{(1)}$. Acerca da prática de atividade física, os alunos foram considerados ativos Quando se exercitavam, ao menos três vezes por semana durante 30 minutos $^{(12)}$.

A análise dos demais dados deu-se com o auxílio do software SPSS versão 16.0. Para averiguar a normalidade e homocedasticidade dos dados empregou-se o Teste de Kolmogorov-Smirnov e de Levene, respectivamente ${ }^{(13)}$. Na análise das variáveis empregouse o Teste de Mann-Whitney e Qui-Quadrado, atendo-se aos cuidados com a homocedasticidade e variânça. Em todos os testes fixou-se um nível de significância de $5 \%$.

O estudo foi conduzido a partir da aprovação do Comitê de Ética em Pesquisa com Seres Humanos da Universidade Federal do Ceará, conforme o protocolo 44/07. Foi exigida, de todos os participantes, a apresentação de termo de consentimento livre e esclarecido assinado pelos seus respectivos pais ou responsáveis.

\section{RESULTADOS}

Conforme as condições sociodemográficas dos 794 adolescentes investigados, observou-se maior adesão feminina: $57,3 \%$ dos avaliados. Por sua vez, na Questão da faixa etária, percebeu-se maior participação dos adolescentes mais jovens, no momento em Que $41 \%$ e $34 \%$ encontravam-se, respectivamente, no intervalo de 14-15 e 12-13 anos (pré-adolescentes). Como observado, em média os investigados tinham 14,3 anos de idade. Além disso, boa parte deles, $40 \%$, cursavam entre $9^{\circ}$ do ensino fundamental e $1^{\circ}$ ano do ensino médio escolar.

No tocante à Questão da renda familiar dos jovens, aQui pesquisada com base no valor do salário mínimo mensal, segundo os resultados revelaram, em sua grande maioria, eles viviam em famílias com 0-3(43,7\%) ou 4-6(28,5\%) salários mínimos mensais, enQuanto outros contavam com renda superior a 10 salários mínimos, condição verificada apenas em 8,5\% dos adolescentes. Mesmo assim, a média da renda mensal, de toda amostra estudada, foi de 15,1 salários. Entretanto, 10\% dos pesquisados não responderam essa indagação.

A maioria dos alunos realiza três $(45,4 \%)$ ou Quarto $(28,5 \%)$ refeições diárias, outro dado bastante preocupante é o fato de três por cento fazer uma única refeição ao dia $(p<0,001)$. Em torno do estado nutricional, os resultados verificados destacam a supremacia dos jovens eutróficos (76\%), em detrimento daqueles em situação de excesso de peso (sobrepeso/obesidade), Que foram aproximadamente $25 \%$ da amostra, e dividiram-se em sobrepeso (20\%) ou obesidade (4\%).

Ao se associar os achados do IMC com o hábito de assistir tevê durante as refeições constatou-se o seguinte: o excesso de peso foi maior e naqueles que mantinham tal hábito em seu cotidiano, os casos de obesidade eram duplicados $(p=0,000)$. Os casos de sobrepeso também foram duplicados entre os pré-adolescentes, 12-13 anos de idade, em relação às demais faixas etárias $(p=$ 0,042 ). Não foi identificado diferenças significativas ao se relacionar o IMC com algumas variáveis como gênero, escolaridade e glicemia capilar. Em contrapartida, por volta de $28 \%$ dos jovens Que praticavam alguma atividade física regularmente apresentavam excesso de peso. Dessa forma, contraditoriamente, as situações de acúmulo de peso corpóreo ocorreram mais entre os adolescentes ativos $(p<0,001)$ (Tabela 2). 
Tabela 2. Distribuição dos adolescentes de escolas particulares, segundo a relação entre o IMC sexo, idade, escolaridade, renda, assistir tevê, glicemia e a prática de atividade física. Fortaleza-CE. 2007 .

\begin{tabular}{|c|c|c|c|c|c|c|c|c|}
\hline \multirow{3}{*}{\multicolumn{2}{|c|}{ Variáveis }} & \multicolumn{6}{|c|}{ Classificação do IMC } & \multirow{3}{*}{$p$} \\
\hline & & \multicolumn{2}{|c|}{ Normal } & \multicolumn{2}{|c|}{ Sobrepeso } & \multicolumn{2}{|c|}{ Obesidade } & \\
\hline & & $\mathrm{n}$ & $\%$ & $\mathrm{n}$ & $\%$ & $\mathrm{n}$ & $\%$ & \\
\hline \multirow[t]{2}{*}{ Sexo } & \multirow{2}{*}{$\begin{array}{l}\text { Feminino } \\
\text { Masculino }\end{array}$} & 354 & 77,8 & 84 & 18,5 & 17 & 3,7 & \multirow[b]{2}{*}{$0,244 *$} \\
\hline & & 247 & 73,7 & 74 & 22,1 & 18 & 5,4 & \\
\hline \multicolumn{9}{|c|}{ Idade (anos) } \\
\hline & $12-13$ & 196 & 72,3 & 64 & 23,6 & 11 & 4,1 & \multirow{3}{*}{$0,042 * *$} \\
\hline \multirow{2}{*}{\multicolumn{2}{|c|}{$\begin{array}{l}14-15 \\
16-17\end{array}$}} & 248 & 76,8 & 60 & 18,6 & 15 & 4,6 & \\
\hline & & 157 & 78,5 & 34 & 17,0 & 09 & 4,5 & \\
\hline \multicolumn{8}{|c|}{ Escolaridade (série) } & \multirow{4}{*}{$0,086^{*}$} \\
\hline & $6-8^{a}$ & 203 & 73,8 & 59 & 21,5 & 13 & 4,7 & \\
\hline \multirow{2}{*}{\multicolumn{2}{|c|}{$\begin{array}{l}9-1^{a} \mathrm{EM} \\
2-3^{\mathrm{a}} \mathrm{EM}\end{array}$}} & 242 & 76,3 & 60 & 18,9 & 15 & 4,8 & \\
\hline & & 156 & 77,2 & 39 & 19,3 & 07 & 3,5 & \\
\hline \multicolumn{9}{|c|}{ Renda mensal (SM) } \\
\hline & $0-3$ & 271 & 78,1 & 64 & 18,4 & 12 & 3,5 & \multirow{4}{*}{$0,659 *$} \\
\hline & $4-6$ & 164 & 72,5 & 51 & 22,6 & 11 & 4,9 & \\
\hline & $7-9$ & 56 & 76,7 & 14 & 19,2 & 03 & 4,1 & \\
\hline & $>10$ & 49 & 72,1 & 15 & 22,1 & 04 & 5,9 & \\
\hline \multicolumn{9}{|c|}{ Assiste tevê enQuanto come } \\
\hline & Sim & 439 & 79 & 93 & 17 & 23 & 04 & \multirow{2}{*}{$0,000 *$} \\
\hline \multicolumn{2}{|r|}{ Não } & 162 & 27 & 64 & 40,8 & 12 & 32,2 & \\
\hline \multicolumn{9}{|c|}{ Glicemia } \\
\hline \multirow{2}{*}{\multicolumn{2}{|c|}{$\begin{array}{l}\text { Normal } \\
\text { Duvidosa }\end{array}$}} & 573 & 76 & 147 & 19,4 & 34 & 4,6 & \multirow{2}{*}{$0,204 *$} \\
\hline & & 28 & 70 & 11 & 27,5 & 1 & 2,5 & \\
\hline \multicolumn{9}{|c|}{ Prática de atividade física } \\
\hline & Ativo & 208 & 72,5 & 63 & 22,0 & 16 & 5,5 & \multirow[t]{2}{*}{$<0,001 * *$} \\
\hline & Sedentário & 393 & 77,5 & 95 & 18,7 & 19 & 3,8 & \\
\hline
\end{tabular}

Legenda: *Teste do Qui-Quadrado; EM= Ensino Médio; ** Teste Mann-Whitney; SM= Salário Mínimo (R\$350,00 reais).

Todos os percentuais dos fatores supracitados foram calculados com base na amostra total, sendo Que a prevalência de sobrepeso ou obesidade seguiu o comportamento geral, ficando em torno de $20 \%$ e $4 \%$, respectivamente.

\section{DISCUSSÃO}

O mundo inteiro têm se preocupado em estudar casos de excesso de peso entre crianças e adolescentes. Uma pesQuisa de abrangência mundial, realizada a partir de dados nacionais de nutrição de diferentes períodos no Brasil, Estados Unidos, China e Rússia, avaliou o excesso de peso em crianças e adolescentes na faixa etária de 6-18 anos. Segundo os resultados revelaram, a prevalência de sobrepeso no Brasil ascendeu de 4,1\% para 13,9\%. O mesmo ocorreu na China (de 6,4\% para 7,7\%) e nos Estados Unidos (de 15,4\% para 25,6\%), enQuanto na Rússia houve redução de 15,6\% para $9,0 \%{ }^{(14)}$.

Em pesquisa brasileira, consoante ressaltado, as prevalências de sobrepeso e obesidade em adolescentes são inferiores às apresentadas em inquéritos de outros países $(7,7 \%)$, e isso corresponde a três vezes menos Que as prevalências encontradas nos Estados Unidos e na Arábia Saudita. Todavia, de acordo com o enfatizado anteriormente, esse Quadro é dinâmico e no Brasil, o excesso de peso entre adolescentes cresce de forma alarmante, em decorrência da transição nutricional Que o país e os adolescentes vivem $^{(4)}$.

Ao se relacionar os resultados desta pesquisa aos últimos estudos do tema em Fortaleza-Brasil, é válido destacar duas pesquisas. A primeira, realizada por Silva (2006) com 720 estudantes, entre 14-19 anos de idade, de 12 escolas públicas identificou percentuais de $10,3 \%$ e $2,6 \%$ para, respectivamente, sobrepeso e obesidade, a partir do método de Cole e colaboradores $(2000)^{(5)}$. Já a segunda executada por Campos e autores (2007), investigou 1.158 crianças e adolescentes (10-19 anos) de 12 escolas, sendo seis públicas e seis particulares. Este estudo identificou uma prevalência de 19,5\% de excesso de peso, a partir do método de Must e colaboradores $(1991)^{(6,15)}$.

Dessa maneira, ao se relacionar os resultados desta pesquisa com Silva (2006) Que usou o mesmo método de classificação do IMC deste estudo, mas em escola pública, ficou evidente Que os casos de sobrepeso e obesidade foram duas vezes maiores Que o encontrado por Silva (2006). Já em torno da investigação de Campos 
e autores (2007) fica difícil tecer comparações, pois os autores estudaram em conjunto crianças e adolescentes e utilizou uma classificação de sobrepeso/obesidade, diferente da nossa Que dividiu em sobrepeso e obesidade ${ }^{(5-6)}$.

A divergência acerca dessas taxas de prevalência em pesQuisa sobre sobrepeso e obesidade infantil e juvenil é reflexo da variabilidade ou discordância dos sistemas de classificação para sobrepeso/obesidade em crianças e adolescentes, motivo de dificuldade na comparação entre estudos dessa temática e da identificação de valores comuns ${ }^{(16)}$.

Um ponto importante de discordância são as ponderações entre estado nutricional e gênero. Quanto à relação de sobrepeso/ obesidade e gênero, ainda há controvérsias no referente a um acometimento maior de um dos sexos. Determinados autores descrevem maior prevalência entre as meninas ${ }^{(4)}$, meninos ${ }^{(17)} \mathrm{e}$ ausência de associação entre sobrepeso/obesidade e gênero ${ }^{(5-6,18)}$.

Apesar das prerrogativas biológicas, na compreensão do fenômeno da ascensão da obesidade como problemática de saúde coletiva, a Organização Pan-Americana da Saúde (OPAS) estima Que $66 \%$ da variação do peso é influenciada pelo ambiente. Os principais fatores desencadeadores estão ligados às mudanças no estilo de vida e nos hábitos alimentares. O consumo exagerado de alimentos com alta densidade energética, somado à diminuição na prática de exercícios físicos são os dois principais motivos e estão diretamente associados à essa epidemia tanto em adultos como em adolescentes ${ }^{(8,19-20)}$

De modo geral, independente do IMC, o jovens possuem importantes taxas de sedentarismo. Contudo, para explicar, por exemplo, o predomínio de casos de excesso de peso entre os alunos ativos neste estudo, levantam-se algumas considerações. Primeiro, um número menor dos pesquisados apresentou sobrepeso/ obesidade, em detrimento dos eutróficos, assim QualQuer opção de atividade física identificada eQuivaleu um percentual considerável para esse grupo. Segundo, tanto esse estudo como a maior parte das pesquisas anteriores, mensurou a atividade física juvenil apenas a partir das ações realizadas durante o lazer desses jovens. Dessa forma, tarefas de considerável dispêndio energético, como transporte ativo no seu deslocamento, atividades domésticas e até profissionais foram preteridas, tornando maior o número de sedentários.

A prática de atividade física é um sólido determinante das características físicas dos jovens. Quantidades excessivas de tempo dedicado a atividades sedentárias são consideradas fatores de risco para a obesidade em jovens, Que acarreta um aumento de $2 \%$ na prevalência dessa patologia para cada hora adicional de tevê na rotina dos jovens ${ }^{(21)}$. Estudo detectou uma chance maior de excesso de peso de até $81 \%$ para meninos Que assistem tevê na maior parte do seu tempo livre(22).

Uma das limitações desta pesquisa foi não contemplar e associar o estado nutricional desses jovens a outros comportamentos como o uso de vídeo games e computador, Que cada vez mais ocupam o tempo juvenil ocioso. Dessa maneira, a execução de outros estudos, transversais e/ou longitudinais é pertinente para clarificar Quais os principais comportamentos e escolhas que cercam os adolescentes acima do peso.

Por fim, torna-se prioritário o estabelecimento de estratégias precoces de prevenção, sobretudo entre a população infanto-juvenil, Quando se leva em conta Que, uma vez instalada, o acúmulo de peso ocasiona alterações fisiopatológicas associadas passíveis de comprometer grande parte do organismo, dificultando ainda mais seu tratamento e acarretando morbidade e Queda na Qualidade de vida do indivíduo.

\section{CONCLUSÕES}

A pesquisa detectou valores importantes em torno do excesso de peso Quando associado a outras pesQuisas desenvolvidas na temática da obesidade juvenil. Estas, ao se somar as investigações realizadas em Fortaleza-Brasil vem levantar a necessidade de se refletir e explorar mais essa problemática, amplamente detectada, no cenário epidemiológico brasileiro. Contudo, não bastam apenas as informações sobre a prevalência de obesidade. São também necessárias ações de intervenção para combater os casos detectados, assim como ações de prevenção, pois o excesso de peso é um dos principais preditores de Qualidade de vida e de saúde.

\section{REFERÊNCIAS}

1. Ebbeling CB, Pawlak DB, Ludwing DS. Childhood obesity: public-health crisis, common sense cure. Lancet 2002; 360:473-482.

2. Grillo MFF, Gorini MIPC. Caracterização de pessoas com Diabetes Mellitus tipo 2. Rev Bras Enferm 2007; 60(I): 4954.

3. World Health Organization. Obesity: Preventing and managing the global epidemic. Report of a WHO consultation on Obesity. Geneva; 1998. p.17-36

4. Neutzling MB, Taddei JA, Rodrigues EM, Sigulem DM. Overweight and obesity in brazilian adolescents. Int I Obes Relat Metab Disord 2000; 24: 869-74.

5. Silva ARV. Investigação dos fatores de risco para diabetes mellitus tipo 2 em adolescentes escolares de Fortaleza-CE [dissertação]. Fortaleza: Departamento de Enfermagem da Universidade Federal do Ceará; 2006.

6. Campos LA, Leite AJM, Almeida PC. Prevalência de sobrepeso e obesidade em adolescentes do município de Fortaleza, Brasil. Rev Bras Saúde Mater Infant 2007; 7(2): 183-90.

7. Wyllie R. Obesity in childhood: an overview. Curr Opinion Pediatrics 2005; 17: 632-5.

8. Willkinson KM. Increasing obesity and children and adolescents: na alarming epidemic. IAAPA 2008; 2 I (12): 3 I-6.

9. Duarte AC, Castellani FR. Semiologia Nutricional. I ${ }^{\text {a }}$ ed. Rio de Janeiro: Axcel Books; 2002.

10. Cole TJ, Bellizzi MC, Flegal KM, Dietz WH. Establishing a standard definition for child overweight and obesity worldwide: international survey. BMJ 2000; 320: 1240.

11. Ministério da Saúde (BR). Secretaria de Atenção à Saúde. Caderno de Atenção Básica-Diabetes Mellitus. Brasília: Ministério da Saúde; 2006. 
12. World Health Organization. Report of a WHO. Consultation Group on Obesity. Obesity: preventing and managing the global epidemic. Geneva; 2000.

13. Hair JF, Anderson RE, Tatham RL, Black WC. Análise multivariada de dados. $6^{\mathrm{a}}$ ed. Rio de Janeiro: Bookman; 2005.

14. Wang Y, Monteiro C, Popkin BM. Trends of obesity and underweight in older children and adolescents in the United States, Brazil, China and Russia. Am I Clin Nutr 2002; 75: $971-7$.

15. Must A, Dallal GE, Dietz WH. Reference data for obesity: $85^{\text {th }}$ and $95^{\text {th }}$ percentiles of body mass index (wt/ht2) and triceps skinfold thickness. Am I Clin Nutr. 1991; 53: 839-46.

16. Silva HG, Chiara VL, Barros ME, Rêgo AL, Ferreira A, Pitasi $\mathrm{BA}$, et al. Diagnosing the nutritional status of schoolchildren: a comparison between Brazilian and international criteria. J Pediatr 2008; 84(6): 550-5.

17. Suñe FR, Costa JSD, Olinto MTA, Pattussi MP. Prevalência e fatores associados para sobrepeso e obesidade em escolares de uma cidade no Sul do Brasil. Cad Saúde Pública 2007 .
23(6): 1361-71.

18. Ramírez E, Grijalva-Haro MI, Ponce IÁ, Valencia ME. Prevalencia de sobrepeso y obesidad em el noroeste de México por tres referencias de índice de massa corporal: diferencias em la classificación. ALAN 2006; 56(3): 25 I-6.

19. Aguirre P. Socioanthropological aspects of obesity in poverty. In: Pan American Health Organization. Obesity and poverty: a new public health challenge. Washington: PAHO; 2000. p. II 22 ,

20. Silva ARV, Damasceno MMC, Marinho NBP, Almeida LS, Araújo MFM, Almeida PC, et al . Hábitos alimentares de adolescentes de escolas públicas de Fortaleza, CE, Brasil. Rev Bras Enferm 2009; 62(1): 18-24.

21. Gambardella AMD, Bismarck-Nasi EM. Televisão e predisposição á obesidade em adolescentes. Rev Paul Pediatr 2000; 18(1): 18-21.

22. Silva KS, Lopes AS, Silva FM. Comportamentos sedentários associados ao excesso de peso corporal. Rev Bras Educ Física Esportes 2007; 2 I (2): 135-41. 